\title{
SIMQUA : un logiciel de simulation de la qualité du bois
}

\author{
JM Leban *, G Duchanois
}

INRA, station de recherches sur la qualité des bois, 54280 Champenoux, France

(Reçu le 5 septembre 1989; accepté le 12 avril 1990)

\begin{abstract}
Résumé - À partir des données morphologiques d'une grume (forme extérieure, nature de la branchaison, taille des accroissements annuels) et des caractéristiques physiques et macroscopiques du matériau telles que la densité du bois et l'angle du fil (fibre torse), nous avons développé un logiciel qui permet la simulation d'un débit scié dont nous pouvons décrire la qualité d'aspect des 4 faces et calculer sa masse volumique. Ce logiciel ouvre des perspectives intéressantes pour l'évaluation objective de l'influence des conditions de croissance d'un arbre ou d'un peuplement sur la qualité du bois produit.
\end{abstract}

simulation / qualité / densité du bois / classement / SIMQUA

Summary - Modelling wood quality. New software : SIMQUA. Forest management requires an understanding of the effect that silvicultural treatments have on wood properties. The forest manager must know the wood quality produced by different silvicultural treatments in order to be able to choose the right one. A variety of software models the effects of cultural practices and environmental factors on the growth and yield of trees (eg Douglas fir); we decided to create software for modelling wood quality with a view to linking it with different growth models. In this work the following aspects of wood quality have been selected: size and distribution of the branches (which are described by whorl, each branch being alive, dead or pruned), grain angle, ring width and pith position at different levels in the tree. The specific gravity can be defined for each ring. According to the model developed the log can be defined with extreme accuracy. The mathematical functions used are the Lagrange polynomial of degree $n-1$ (useful in the finite element method). It is now possible to simulate the sawing of boards from the described log and to compute the position, size and shape of knots on the 4 faces of each board and the specific gravity of each board. These results permit us to simulate different visual rules to estimate the quality of the sawn logs. In future it will be possible to optimize the sawing by visual or mechanical criteria. The data needed by this software (ring width, pith position, description of the branches) can be measured or produced by a growth model. The models is able to predict specific gravity variation in the tree and the angle and diameter of the branches in the different whorls must be produced before our new tool totally efficient.

modelling / wood quality / wood density / grading / SIMQUA

\footnotetext{
* Correspondance et tirés à part
} 


\section{INTRODUCTION}

Toute gestion de production passe par la maîtrise des coûts d'exploitation. Cette façon de procéder, connue de tous et courante dans l'industrie est toutefois difficile à mettre en œuvre lorsque les outils de production sont mal connus. C'est le cas de la production ligneuse pour laquelle 2 types de difficultés majeures existent:

- la grande variabilité des propriétés des produits inhérente à toute production biologique ;

- la durée de production, obstacle majeur à toute démarche empirique dès lors que I'on envisage de produire autrement.

Cependant, les prix des produits ligneux existent et sont établis par le marché pour partie et par des règles de classement (sciages pour l'ébénisterie, la menuiserie, la structure par exemple). Ces règles de classement sont souvent spécifiques à chaque pays producteur de bois et elles s'efforcent de pénaliser le moins possible la ressource nationale. Suivant les cas, les caractéristiques évaluées sont la nodosité, les propriétés mécaniques, l'angle du fil, les poches de résine.

Le besoin d'outils de gestion de production ou de gestion sylvicole est donc évident et plus particulièrement le besoin d'outils d'évaluation de la qualité ou du classement des produits d'un arbre issu d'un traitement sylvicole donné ou d'une population d'arbres (évaluation de la qualité d'une ressource). En effet, comment justifier des investissements tels que l'étalage, les éclaircies, sans être en mesure d'en évaluer la plus-value qui en résultera?

L'objet de cet article est de présenter un nouveau logiciel destiné à modéliser la qualité des produits sciés tirés d'une grume. Après un inventaire des outils ou approches déjà existants, nous indique- rons d'abord les descripteurs de l'arbre qui constituent les entrées de cet outil informatique. Nous poursuivrons en présentant les sorties actuellement fonctionnelles du logiciel. Avant de conclure sur les perspectives de développement de cet outil, nous indiquerons les données dont nous disposons pour l'alimenter ou à défaut la démarche poursuivie pour les acquérir.

\section{Les approches et outils déjà existants}

La prise en compte de caractéristiques morphologiques des arbres pour évaluer leur qualité a été abordée dans des travaux que l'on peut classer en 3 familles.

\section{Les études globales}

En Amérique du Nord, un travail de modélisation globale associant plusieurs équipes est poursuivi actuellement. II a pour but de rassembler des données obtenues séparément dans une série de logiciels qui décrivent toute la chaîne de production du douglas de reboisement (Mitchell et al, 1982; Mitchel, 1988; Bergstrom, 1988):

- modèles de croissance, arbre et peuplements;

- modèle de dégradation biologique (interactif avec le modèle de croissance);

- modèle de sciage avec description de la nodosité et surtout optimisation de débit;

- modèle d'analyse financière.

Cette approche est très intéressante par son aspect global, mais il semble que les caractéristiques de qualité des produits sont définies de manière assez approximative. En effet, l'évaluation du classement visuel résulte de relations statistiques établies entre des débits réels provenant d'arbres issus de traitements sylvicoles 
particuliers. Cette méthode, qui est coûteuse, ne prend pas en compte la démarche d'optimisation de la qualité puisque les débits sont de dimensions constantes quel que soit le traitement sylvicole.

\section{L'exploitation statistique des données d'inventaire forestier}

En Finlande, Karkkainen (1986a et b) a proposé une démarche originale qui permet d'exploiter les données de l'inventaire forestier national pour définir de façon moyenne, par essence et pour des arbres types, des zones sans nœuds, avec nœuds morts et avec nœuds vivants (pin sylvestre, épicéa et bouleau). A partir de ces donnée, il est possible d'optimiser le tronçonnage en forêt. La géométrie extérieure des arbres étant connue, un logiciel de simulation de débits évalue les produits obtenus. Ces résultats ne sont pas transposables en France, compte tenu des conditions particulières de production propres à la Finlande.

Un article finlandais récent de Vaïsänen et al (1989) propose un modèle de simulation de la qualité des sciages qui prend en compte différentes conditions de production sylvicoles. Ce modèle, établi pour le pin sylvestre, fait la synthèse des connaissances établies de longue date sur la branchaison de cette essence en fonction de différentes conditions de sylviculture dont l'élagage. L'étude du problème est faite en 5 étapes qui sont:

- la croissance de l'arbre (proportionnelle à la photosynthèse);

- la croissance et la structure du houppier (description de la branchaison);

- la qualité des billons (forme des billons, diamètre des branches);

- la qualité des sciages;

- le classement des sciages.
Ce modèle est original par la simulation de l'élagage naturel qui semble cependant perfectible, selon les auteurs. Par contre l'angle des branches est pris constant, ce qui minimise la surface de nœuds (lorsque l'angle est proche de $90^{\circ}$ ). Enfin la simulation de la qualité des sciages décrit la nodosité des débits "export" qui sont en général des sciages de cœur, ce qui minimise la dimension des nœuds.

\section{La simulation de débit}

Les récentes évolutions technologiques dans le domaine de la visualisation des défauts internes d'une grume (tomographie industrielle, micro-ondes) permettent d'envisager, en scierie, l'emploi de logiciels d'optimisation de débits qui utilisent ces données (position et type de défaut) comme critères de choix des produits $(\mathrm{Oc}$ cena et Tarchoco, 1988). Dans ce cas particulier, aucune connexion avec l'amont, c'est-à-dire la croissance, n'est envisagée.

L'outil proposé ici est autonome, connectable avec des logiciels amont (simulation de croissance) et aval (optimisation de débit) et capable de simuler avec une bonne précision et de manière fonctionnelle les caractéristiques visuelles et mécaniques des débits.

\section{L'introduction des données géométriques}

La grume est décrite par sections (plan radial-tangentiel). Le nombre de sections est fonction du niveau d'information disponible (par exemple, 2 sections si la grume est supposée d'axe rectiligne et plus si on a la possibilité de décrire sa forme exacte) et définit le niveau d'approximation des caractéristiques de la grume entre chaque section. Pour la simulation d'une grume réelle, si les sections extrêmes sont parfaite- 
ment connues, les données des sections intermédiaires peuvent être estimées de plusieurs façons :

- par des lois générales caractéristiques d'une essence, tirées de l'expérimentation;

- par des mesures sur carottes de sondage pour évaluer quelques arbres;

- en utilisant des résultats de modèles de croissance pour simuler l'impact de différentes pratiques sylvicoles.

Les données introduites sont géométriques et qualitatives. Pour chaque section de la grume, nous décrivons la géométrie des cernes annuels et pour chaque verticille, nous donnons les caractéristiques des branches qui le composent (position en hauteur, angle d'insertion, diamètre, état sanitaire).

\section{Les données géométriques par section}

\section{La position du centre de la section}

Elle est définie dans un repère de référence qui est la section de base de la grume.

\section{Le diamètre de la section}

Les sections et les cernes annuels sont supposés circulaires de manière à limiter le nombre de données à introduire.

\section{La largeur et nombre de cernes dans la section}

Ces données sont définies pour un rayon et lépaisseur de l'écorce est assimilée à un cerne supplémentaire. La largeur de chaque cerne est exprimée en terme de pourcentage du rayon de la section.

\section{La position de la moelle dans la section}

Elle peut être choisie excentrée, ce qui est fréquemment rencontré. Dans ce cas elle peut présenter une courbure spatiale différente de celle de la tige.
Si la moelle est excentrée tous les centres des cercles décrivant les cernes annuels sont placés sur le segment MO de la figure 1. Leur position sera obtenue de la façon suivante :

$$
\frac{M L_{i}}{M E}=\frac{M C_{i}}{M O}
$$

$M$ : moelle

$L_{i}$ : limite du cerne $i$

$E$ : écorce

$C_{i}$ : centre du cercle décrivant le cerne $i$

$O$ : centre du cercle extérieur

\section{Formulation mathématique}

Chaque grandeur définie ci-dessus est estimée par une approximation nodale utilisant le polynôme de Lagrange de degré $n$ 1 ( $n$, nombre de sections) tel que:

$G=N_{j}(\lambda) G_{i}, i=1, n$ avec sommation sur $i$

$G$ est la grandeur à estimer;

$N_{i}(\lambda)$ sont les fonctions de l'approximation du type :

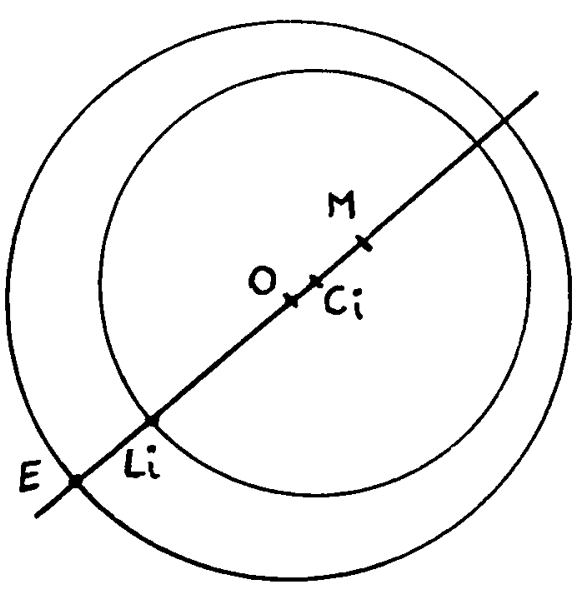

Fig 1. Section avec moelle excentrée. 


$$
N_{i}(\lambda)=\prod_{\substack{j=1, n \\ i \neq j}} \frac{\left(\lambda_{j}-\lambda\right)}{\left(\lambda_{j}-d_{i}\right)}
$$

$\lambda$ est un paramètre dont les valeurs particulières sont choisies arbitrairement de manière à satisfaire les relations suivantes :

$$
\begin{array}{llll}
N_{i}\left(\lambda_{j}\right)=\delta_{i j} & \delta_{i j}=0 & \text { si } & i \neq j \\
\Sigma N_{i}(\lambda)=1 & \delta_{i j}=1 & \text { si } & i=j
\end{array}
$$

Chaque grandeur est définie en fonction de ses valeurs particulières (données introduites). Les fonctions $N_{i}(\lambda)$ sont indépendantes de la grandeur et peuvent être prises identiques pour l'ensemble des approximations des données géométriques. Une section est donc entièrement caractérisée par une valeur particulière de $\lambda$, qui dans notre cas peut être liée à la hauteur dans la grume.

\section{Les caractéristiques de branchaison}

Les branches sont associées à un verticille et décrites par un tronc de cône puis positionnées de la manière suivante :

- position en hauteur dans la grume du point d'intersection de la moelle de la branche avec la moelle du tronc;

- angle de l'axe de la branche avec la moelle du tronc (plan longitudinal-radial);

- angle dans la section (plan radialtangentiel);

- diamètre de sortie de la branche (sur l'écorce);

- définition de l'âge à partir duquel la branche s'est désséchée;

- si la branche a été élaguée, définition de l'âge de l'élagage.

Nous avons pu vérifier que les hypothèses de forme des branches sont satis- faisantes pour l'épicéa (axe de la branche linéaire, section transversale circulaire, section longitudinale conique).

\section{La formulation mathématique pour la branchaison}

Les paramètres définis ci-dessus autorisent une orientation correcte du tronc de cône qui décrit chaque branche. Les équations donnant cette description géométrique sont identiques aux précédentes (approximation nodale).

L'axe du cône est défini par 2 points :

- le point de départ de la moelle

$$
x_{1}\left(x_{1}, y_{1}, z_{1}\right)
$$

- le point de sortie de la grume

$$
x_{2}\left(x_{2}, y_{2}, z_{2}\right)
$$

Un point particulier de l'axe est décrit par

$$
X=N_{i}(\lambda) X_{i} \quad \text { avec } X(x, y, z)
$$

Pour une droite nous avons :

$$
\begin{array}{ll}
N_{1}=1-\lambda & \\
N_{2}=\lambda & \text { si } O<\lambda<1 \\
X=(1-\lambda) X_{1}+\lambda X_{2} &
\end{array}
$$

De même pour le rayon :

$$
R=N_{i}(\lambda) R_{i}=\lambda R_{2}
$$

car $R_{1}$, rayon du début de la branche est nul

Le critère sanitaire et l'élagage se traduisent simplement par une valeur particulière de $\lambda$.

\section{Le fonctionnement du logiciel SIMQUA}

\section{La visualisation \\ des données introduites}

Le traitement du fichier de données permet la représentation graphique de la grume 
étudiée. Sur la $1^{\text {re }}$ photographie d'écran (fig 2) nous voyons :

- une coupe transversale passant par la moelle (partie gauche de l'écran);

- une vue de dessus (partie supérieure droite de l'écran);

- la représentation d'une section quelconque de la grume. Sur cette vue, on distingue une zone juvénile, adulte, l'aubier (pour les essences concernées) et l'écorce. La taille de ces différentes zones est déterminée par les données introduites.

Sur la figure 3 , nous visualisons les données concernant la branchaison :

- sur la partie de gauche de l'écran, la surimpression sombre visualise l'étendue d'un verticille;

- sur la partie inférieure droite de l'écran, nous représentons la répartition angulaire des branches du verticille. Pour chaque branche, nous voyons une zone adhérente, non adhérente et une longueur élaguée;

- la partie supérieure droite de l'écran montre l'angle que font toutes les branches par rapport à la moelle. Les branches sont donc toutes ramenées dans le même plan.

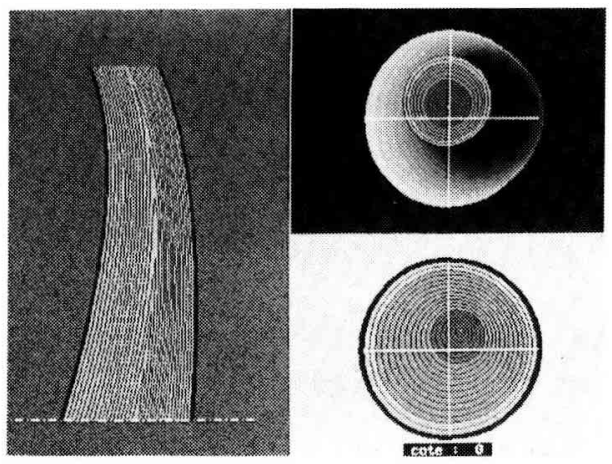

Fig 2. Description de la grume.
Ces 2 premiers écrans sont destinés au contrôle visuel des données introduites et à l'illustration graphique du logiciel. Ce ne sont pas des étapes indispensables au fonctionnement.

Par contre dans la version du logiciel présentée ici, l'écran représenté sur la figure 4 est fonctionnel puisqu'il permet de choisir et de positionner le débit que l'on souhaite réaliser.

\section{L'exploitation des résultats}

Sur la figure 5 sont représentées les 4 faces du débit choisi. II est possible d'exploiter les caractéristiques dimensionnelles des nœuds.

\section{Le classement d'aspect}

\section{Les noeuds}

À l'aide d'un traitement informatique approprié, nous obtenons le volume de nœud contenu dans le débit; en particulier pour l'intersection face du débit branches, il est possible de calculer pour chaque nceud les caractéristiques suivantes :

- forme et surface du nœud;

- position dans la face du débit;

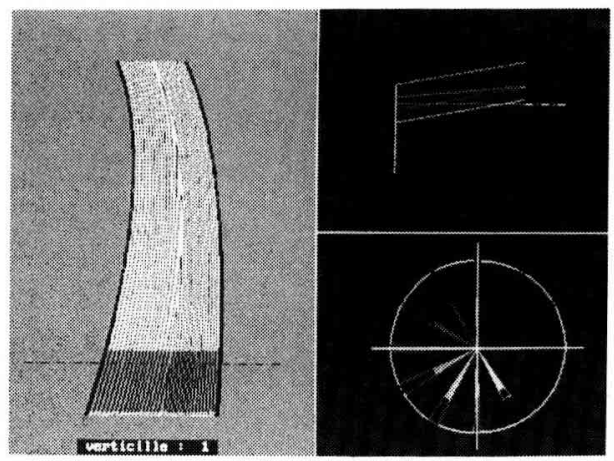

Fig 3. Description des branches. 


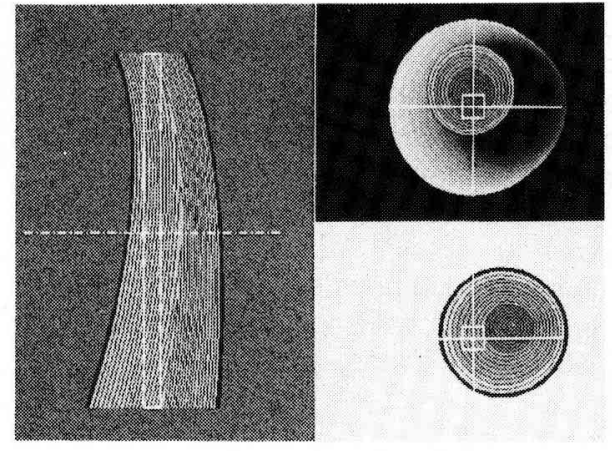

Fig 4. Définition du débit.

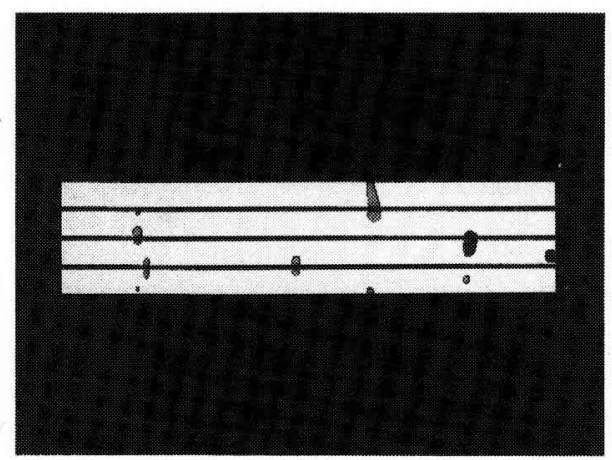

Fig 5. Vue des nœuds sur les 4 faces du débit.

- état sanitaire (sain, mort et éventuellement altéré).

\section{Les flaches}

D'une manière analogue, nous calculons pour chaque face du débit la forme et la position des flaches (intersection débit grume).

Les résultats de ces calculs sont donc disponibles sous forme de fichiers, traités ensuite en fonction des applications souhaitées (un classement normalisé par exemple).

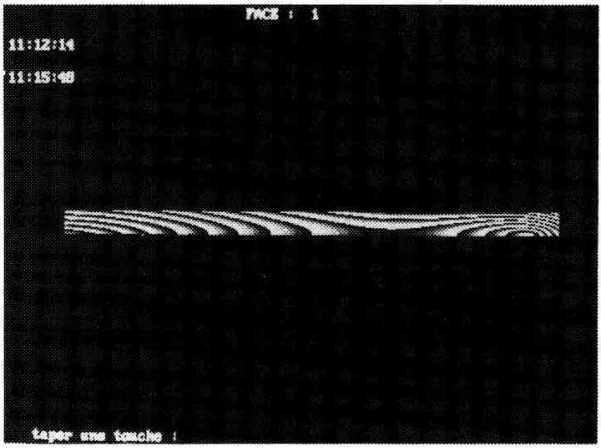

Fig 6. Vue de l'aspect esthétique sur une face.

\section{La densité du bois}

Nous pouvons décrire une variation locale de densité du bois, c'est-à-dire introduire pour chaque cerne la variation fine de densité et ceci pour tous les rayons d'une même section dans la mesure où ce caractère varie, ce qui arrive lorsque la moelle est excentrée. Actuellement, nous faisons varier la densité à l'intérieur d'un cerne de façon linéaire. La densité du bois des branches sera prise constante en première approche. Sur la figure 6 , nous voyons la représentation, sur une face, de cette variation de densité, ce qui donne l'aspect du débit. Le résultat numérique est la densité moyenne du débit. Cette valeur pourra être exploitée lorsque des données réalistes de densité seront introduites (voir p 490).

\section{L'angle du fil}

C'est une caractéristique importante du matériau qui influe en particulier sur les déformations au séchage et sur la qualité des surfaces usinées. L'angle du fil a 2 composantes :

- la première, dans le plan radiallongitudinal, est donnée par la décroissance du diamètre; 
- la seconde (fibre torse) est mesurée dans le plan tangentiel-longitudinal et peut être introduite pour plusieurs hauteurs dans l'arbre.

C'est cette dernière caractéristique qui est prise constante par le logiciel. Dans un premier temps ne sera pas prise en compte la variation d'angle du fil à proximité des nœuds, ceci faisant l'objet d'une étude spécifique (voir p 491). De même que pour la densité cette valeur sera exploitable lorsque nous aurons introduit des données réalistes.

\section{La démarche envisagée pour un usage réaliste de SIMQUA}

Dans sa forme actuelle, le logiciel simqua est donc capable de simuler la nodosité, la distribution de densité et d'angle du fil du bois de toute pièce tirée de n'importe quel arbre. Pourvu de ces données élémentaires décrivant la pièce en tout point, il est en principe possible, sous réserve d'un investissement en caractérisation mécanique et en calcul numérique, de prévoir le comportement et l'aptitude technologique du débit en question ou plus prosaïquement son classement visuel et mécanique (voir p 491).

L'utilisation de SIMQUA avec des modèles de simulation de croissance, c'est-àdire pour prédire de manière légère la qualité des débits tirés d'un arbre, d'arbres représentatifs d'un peuplement ou d'une ressource, n'est pas encore réaliste. En effet, nous avons besoin actuellement d'un nombre d'entrée très détaillée qu'il n'est pas envisageable de collecter.

C'est pourquoi nous nous proposons d'introduire dans SIMQUA des lois ou relation statistiques établies ou à établir entre un ensemble de descripteurs pertinents des arbres et les données intensives actuellement nécessaires. Ceci devrait nous permettre d'utiliser SIMQUA, dûment pourvu de ces relations statistiques, à partir d'un ensemble minimal de descripteurs. Dans ce qui suit nous présentons la méthodologie que nous avons mise en œuvre ou que nous envisageons d'adopter pour établir ces relations.

\section{Les données géométriques et morphologiques}

\section{Les largeurs de cerne}

La connaissance des distributions de largeurs de cerne dans une pièce de bois est un élément déterminant de ses aptitudes technologiques. Ces données caractéristiques des conditions de croissances peuvent être fournies par des logiciels de simulation de croissance qui sont opérationnels (Ottorini, communication personnelle). C'est à partir de ces données qu'il sera possible de caractériser pour une bonne part les propriétés physiques des produits issus de chaque grume (voir p 491).

\section{La branchaison}

Nous avons entrepris une importante campagne de mesures afin de pouvoir disposer rapidement de modèles statistiques qui permettent de décrire les branches principales et secondaires de l'épicéa commun (diamètre, nombre de branches par verticille, répartition verticale, angulaire dans le plan transversal [fig 3]) en fonction de la position de l'arbre. Ces caractéristiques doivent être acquises pour chaque branche et surtout pour la partie incluse dans le tronc, ce qui implique un important travail expérimental. 


\section{Les caractéristiques physiques et mécaniques}

\section{La densité du bois}

De nombreux travaux établissent les relations statistiques entre la densité du bois et la largeur de cerne. Ces relations, établies pour des études de variabilité, ne présentent par un caractère fonctionnel suffisant pour être utilisées directement. II est indispensable de pouvoir modéliser les variations locales de densité à l'intérieur d'un arbre si l'on a l'ambition de prévoir les caractéristiques de différents produits. $\mathrm{Ce}$ travail, en cours de réalisation, a pour objectif final la prédiction fine des propriétés mécaniques du matériau (Leban et de Reboul, 1987; Chantre, 1989). Les relations densité du bois propriétés mécaniques restent à établir pour le bois de compression qui représente un volume de bois important à proximité des nœuds. Cette étude est utile puisque le bois de compression à une densité forte pour des propriétés mécaniques faibles.

\section{L'angle du fil}

Deux types d'étude sont actuellement en cours :

- la variation de l'angle du fil en fonction de la position dans l'arbre. Ce travail, réalisé sur l'épicéa commun a pour objet de caractériser la composante torse de l'angle du fil (voir p 490) pour du bois sans défauts (entre les verticilles).

- La variation de l'angle du fil autour d'un noeud est une caractéristique très importante pour déterminer l'influence des nœuds sur les propriétés mécaniques de pièces de structure. Des travaux préparatoires sont déjà réalisés (Duchanois et al, 1987), mais il reste à mettre au point une technique de mesure des variations tridimensionnelles de l'angle du fil autour des nœuds.

\section{Les perspectives de développement du logiciel SIMQUA}

\section{La simulation des règles de classement}

La première application potentielle du logiciel mis au point est le classement. Pour réaliser cet objectif, il faut écrire un programme de traitement des données (nœuds, flaches) en fonction d'un choix de règles de classement. Dans un premier temps, la simulation des règles de classement établies par le CTBA devrait donner des idées assez précises sur un niveau de valorisation de différents types d'arbres ou de peuplements, voire de la ressource d'une zone géographique. Dans une deuxième étape, nous envisageons de simuler d'autres classements (KAR par exemple) afin de situer objectivement notre ressource par rapport à d'autres marchés. Ce type d'information peut apporter des éléments de négociation pertinents dans le cadre de la CEE.

Par ailleurs une évaluation du rendement matière de la grume complète avec un logiciel d'optimisation de débit pour une série de produits types donnera une évaluation de performances en termes de qualité de la forme de la grume.

\section{Le calcul de la rigidité des débits}

À partir des propriétés mécaniques (déduites de la densité du bois) et de la description de la structure d'un débit (largueur des cernes, angle du fil), il est possible de calculer la rigidité de ces débit. Cette caractéristique dépend de la géométrie du débit (à masse égale et à propriétés mécaniques égales une poutre de section rectangulaire est plus rigide qu'une poutre de section carrée si elle est sollicitée suivant la hauteur de sa section). Ce développement sera particulièrement utile pour réduire les coûts des campagnes de qualifi- 
cation d'une ressource et très puissant puisque le nombre de débits possibles ne sera pas limité du fait du faible coût des simulations.

\section{La simulation d'autres applications}

La technique de simulation que nous utilisons offre différentes possibilités de réalisation de débits. Nous pouvons envisager une application déroulage, tranchage et surtout l'introduction d'autres données de qualité telles que la couleur, les retraits pour simuler, à l'aide d'autres logiciels connectés à SIMQUA, les déformations dues au séchage, l'aspect esthétique des produits obtenus. Tout ceci requiert au préalable l'établissement de relations statistiques, entre une description fine des débits actuellement disponible et les propriétés telles que la rétractabilité et la couleur du bois.

\section{L'optimisation de débits}

C'est probablement une application importante qui sera développée. En effet, nous avons la possibilité d'introduire des contraintes de qualité dans les algorithmes d'optimisation qui utilisent essentiellement dans leur forme actuelle des contraintes géométriques.

\section{CONCLUSIONS}

Le logiciel que nous avons présenté illustre une nouvelle démarche d'approche de la qualité des bois. Le fait de définir un outil de synthèse des connaissances n'est pas une idée originale en soi, mais nous avons montré ici la faisabilité de son utilisation. De plus ce cheminement a eu pour conséquence d'une part, de faire un point des connaissances acquises et à acquérir pour modéliser la qualité des bois et d'autre part, d'ouvrir un champ nouveau d'applications aux travaux que nous avons menés jusqu'à présent. Parmi ces applications nouvelles, la plus importante est la contribution que nous pouvons apporter, à la réalisation d'outils de gestion et d'évaluation d'une ressource forestière. D'autres exemples, tels que l'évaluation objective de la rentabilié de l'élagage ou de l'étude des corrélations juvénile-adulte montrent qu'une approche de ce type ouvre de nouvelles perspectives de recherche pour une meilleure évaluation de la qualité des bois qui pourra être faite à des coûts plus faibles.

D'autres applications sont également envisageables dans les industries de première transformation, en particulier pour des optimisations de débits. Cela implique des techniques de prise d'informations sur grumes qui ne sont pas encore très répandues (scanneurs, caméras, calculateurs rapides).

Nous disposons à présent d'un outil qu'il faut valider et compléter par l'adjonction de données fiables pour les caractères morphologiques et pour les caractéristiques physiques. Ces données devront être obtenues par des modèles statistiques qui seront établis par plusieurs campagnes de mesures d'abord intensives puis extensives. Nous insistons ici sur le fait que nous disposons d'un outil puissant mais relativement vide de données à traiter. C'est donc tout un programme de recherche pluridisciplinaire qui est défini par cette approche nouvelle.

\section{RÉFÉRENCES}

Bergstrom D (1988) Tying wood quality to growth and yield. For Res West USDA For Serv Rep June, 1-5 
Chantre $\mathrm{G}$ (1989) Liaison entre rigidité et densité du bois à l'intérieur d'un cerne. Application au cas de l'épicéa commun (Picea abies Karst). DEA Sci bois INPL Nancy, St de Rech S Qual Bois INRA, Champenoux

Duchanois G, Leban JM, Triboulot P (1988) Concentration de contraintes au voisinage d'un nceud sous sollicitation simple. Congrès de rhéologie du bois. Bordeaux, 8-9 juin, 363-386

Vaïsänen $H$, Kellomaki $S$, Oker-Blom $P$, Valtonen E (1989) Structural development of Pinus sylvestris stands with varying initial density: a preliminary model for quality of sawn timber as affected by silvicultural measures. Scand J For Res 4, 223-238

Karkkainen M (1986a) Model of knottiness of wood material in pine, spruce and birch. Silva Fenn 20, 107-116
Karkkainen M (1986b) Value relations of pine and spruce stems. Silva Fenn 20, 117-127

Leban JM, de Reboul L (1988) Modélisation de la rigidité du bois d'épicéa commun en fonction des paramètres de structure. Congrès de rhéologie du bois. Bordeaux, 8-9 juin, 1-12

Mitchell KJ (1988) Sylver: Modelling the impact of silviculture on yield, lumber value and economic return. For Chron 64, 127-131

Mitchell K, Oswald H, Ottorini JM (1982) Modelling the growth of Douglas fir in France forest growth modelling and simulation. IUFRO Meeting 4-8 October 1982, Vienna, Austria

Occena LG, Tanchoco JMA (1988) Computer graphics simulation of hardwood log sawing. For Prod J 38, 72-76 